\title{
Low-Temperature Sintering Behavior of Aluminum Nitride Ceramics with Added Copper Oxide or Copper
}

\author{
Jin-Geun Hwang, Kyung-Sik Oh, Tai-Joo Chung, Tae-Heui Kim, and Yeong-Kyeun Paek ${ }^{\dagger}$ \\ The Center of Biomedical Materials and Biotechnology, The Center of Green Materials Technology, \\ Department of Materials Science and Engineering, Andong National University, Andong 36729, Korea
}

(Received November 15, 2018; Revised December 3, 2018; Accepted December 3, 2018)

\begin{abstract}
The low-temperature sintering behavior of $\mathrm{AlN}$ was investigated through a conventional method. $\mathrm{CaF}_{2}, \mathrm{CuO}_{\mathrm{O}}$ and $\mathrm{Cu}$ were selected as additives based on their low melting points. When sintered at $1600^{\circ} \mathrm{C}$ for $8 \mathrm{~h}$ in $\mathrm{N}_{2}$ atmosphere, a sample density $>98 \%$ was obtained. The X-ray data indicated that eutectic reactions below $1200^{\circ} \mathrm{C}$ were found. Therefore, the current systems have lower liquid formation temperatures than other systems. The liquid phase showed high dihedral angles at triple grain junctions, indicating that the liquid had poor wettability on the grain surfaces. Eventually, the liquid was likely to vaporize due to the unfavorable wetting condition. As a result, a microstructure with clean grain boundaries was obtained, resulting in higher contiguity between grains. From EDS analysis, oxygen impurity seems to be well removed in AlN lattice. Therefore, it is believed that the current systems are beneficial for reducing sintering temperature and improving oxygen removal.
\end{abstract}

Key words : $\mathrm{AlN}, \mathrm{CaF}_{2}, \mathrm{CuO}, \mathrm{Cu}$, Low-temperature sintering

\section{Introduction}

A IN has various properties such as high thermal conductivity, low dielectric constant, high dielectric breakdown strength, high electrical resistivity, good electrical insulation, high corrosion resistance to halogens and plasma, nontoxicity, chemical inertness and thermal expansion coefficient matching that of silicon. These properties are known to be suitable for applications as substrate and package materials in integrated circuits for electronic devices, electrostatic chuck and plasma etchers for semiconductor manufacturing, heaters for CVD equipment, heat sinks, laser diode heat spreaders, optoelectronic parts, cutting tools, ignition modules, casting crucibles, etc. ${ }^{1-7)}$

From this perspective, AlN is considered to be a very promising material. However, It is difficult to densify by pressureless sintering due to its covalent bonding nature. In order to reduce manufacturing costs and take advantage of the cofiring of multilayer substrates, it is imperative to lower the sintering temperature. To date, sintering additives such as $\mathrm{CaF}_{2}$ as well as rare-earth and alkaline-earth oxides have been used for reducing sintering temperature and achieving a fully dense state. These additives mainly contribute to densification and thermal conductivity. A eutectic liquid is formed by the reaction between the additives and $\mathrm{Al}_{2} \mathrm{O}_{3}$ inherited from $\mathrm{AlN}$ powder itself. ${ }^{5-7)}$ The liquid improves densification by allowing matrix atoms to

\footnotetext{
Corresponding author: Yeong-Kyeun Paek

E-mail : ykpaek@anu.ac.kr

Tel : +82-54-820-5754 Fax : +82-54-820-6211
}

easily move through it. For the densification, thus, liquid formation temperature and liquid characteristics are crucial during sintering.

In general, thermal energy (heat) is transferred in solids by two modes: lattice vibration (phonon) and free electron. ${ }^{8)}$ In $\mathrm{AlN}$, heat is primarily transferred through phonons. Therefore, the thermal conductivity of AlN depends on the factors scattering phonons and thereby reducing the thermal conductivity. The phonons scatter at impurities and structural defects such as vacancies, grain boundaries, and pores. The main impurity in AlN is oxygen. Oxygen dissolved into the AlN matrix exists in the form of $\mathrm{Al}_{2} \mathrm{O}_{3}$. In addition, when $\mathrm{AlN}$ powder is exposed to air, $\mathrm{Al}_{2} \mathrm{O}_{3}$ is spontaneously formed on the surfaces of powder particles. Further, when the $\mathrm{Al}_{2} \mathrm{O}_{3}$ is incorporated into the matrix, aluminum vacancies are produced. For AlN, the aluminum vacancies have a negative effect on improving the thermal conductivity. ${ }^{9-11)}$ Thus, in order to enhance the thermal conductivity, it is important to get rid of oxygen in AlN lattice.

In this context, a successful additive for sintering AlN should concomitantly improve the densification as well as the thermal conductivity even at low sintering temperatures. Therefore, the sintering additives used for AlN should be carefully selected. In order to identify such an additive, $\mathrm{CaF}_{2}, \mathrm{Cu}$ and $\mathrm{CuO}$ were investigated due to their low melting points as well as the oxygen gettering effect. In this study, densification, microstructure evolution, and thermal conductivity were examined for samples sintered at the low temperature of $1600^{\circ} \mathrm{C}$. Based on the results, the present systems showed favorable characteristics for improving the low-temperature sintering behavior of AlN. 


\section{Experimental Procedure}

Pure AlN (Kojundo chemical Co., Japan; purity: 99.9\%) was used as a starting material, as described in Table 1 . A mixed powder composed of $\mathrm{CaF}_{2}, \mathrm{Al}_{2} \mathrm{O}_{3}$, and $\mathrm{CuO}$ or $\mathrm{Cu}$ was added to the AlN as an additive. Two kinds of sample powder were prepared: (1) $86 \mathrm{AlN}-2.8 \mathrm{CaF}_{2}-8.4 \mathrm{Al}_{2} \mathrm{O}_{3}-2.8 \mathrm{Cu}(\mathrm{S} 1)$, and (2) $86 \mathrm{AlN}-2.8 \mathrm{CaF}_{2}-8.4 \mathrm{Al}_{2} \mathrm{O}_{3}-2.8 \mathrm{CuO}(\mathrm{S} 2)$. The compositions were empirically determined and represented in wt.\%.

The mixed powders were prepared by wet-milling for $72 \mathrm{~h}$ in a polyethylene bottle. Alumina balls and ethyl alcohol were used as the milling media and solvent, respectively. After ball milling, the powders were dried in a vacuum oven at $80^{\circ} \mathrm{C}$ for $36 \mathrm{~h}$. Then, the dried powders were crushed and sieved (125 mesh). Each powder of $\sim 1 \mathrm{~g}$ was uniaxially pressed into pellets under $0.5 \mathrm{MPa}$, followed by cold isostatic pressing under $100 \mathrm{MPa}$ for $3 \mathrm{~min}$. The green pellets were about $12 \mathrm{~mm}$ in diameter and $5 \mathrm{~mm}$ high. Given possible eutectic temperatures in between the sample compositions (Table 2), the green compacts were sintered at $1600^{\circ} \mathrm{C}$ for various times after keeping them at $1000^{\circ} \mathrm{C}$ for $2 \mathrm{~h}$ under $\mathrm{N}_{2}$ atmosphere in a graphite resistance furnace. The heating and cooling rates were each approximately $5^{\circ} \mathrm{C} / \mathrm{min}$.

Sintered densities were obtained by the Archimedes method using distilled water as an immersion medium. Every density datum was determined by an average of three similar specimens. Based on the rule of mixture considering the densities of $3.26 \mathrm{~g} / \mathrm{cm}^{3}$ for AlN, $3.18 \mathrm{~g} / \mathrm{cm}^{3}$ for $\mathrm{CaF}_{2}, 3.95 \mathrm{~g} / \mathrm{cm}^{3}$ for $\mathrm{Al}_{2} \mathrm{O}_{3}, 6.31 \mathrm{~g} / \mathrm{cm}^{3}$ for $\mathrm{CuO}$, and $8.96 \mathrm{~g} /$ $\mathrm{cm}^{3}$ for $\mathrm{Cu}$, the theoretical densities of samples $\mathrm{S} 1$ and $\mathrm{S} 2$ are determined to be 3.32 and $3.31 \mathrm{~g} / \mathrm{cm}^{3}$, respectively. Linear shrinkage and weight loss were calculated by the difference in dimension and mass between sintered and green bodies, respectively.

In order to analyze the developed phases of the sintered

Table 1. Impurity Contents of AlN Starting Powder

\begin{tabular}{cc}
\hline Impurity element & Content (\%) \\
\hline $\mathrm{Ca}$ & 0.008 \\
$\mathrm{Fe}$ & $\mathrm{ND}$ \\
$\mathrm{Na}$ & 0.004 \\
$\mathrm{Ni}$ & $\mathrm{ND}$ \\
$\mathrm{Si}$ & 0.001 \\
$\mathrm{O}$ & $\mathrm{NA}$ \\
\hline
\end{tabular}

Table 2. Possible Eutectic Reactions between Compositions

\begin{tabular}{ccc}
\hline Composition & $\begin{array}{c}\text { Eutectic temperature } \\
\left(\mathrm{T}_{\mathrm{E}}\right)\end{array}$ & \\
& $1065^{\circ} \mathrm{C}$ & Cu-related eutectic \\
$\mathrm{Cu}-\mathrm{Cu}_{2} \mathrm{O}$ & $1091^{\circ} \mathrm{C}$ & reactions \\
$\mathrm{Cu}_{2} \mathrm{O}-\mathrm{CuO}$ & $1165^{\circ} \mathrm{C}$ & \\
$\mathrm{CuO}-\mathrm{Al}_{2} \mathrm{O}_{3}$ & $1397^{\circ} \mathrm{C}$ & Ca-related eutectic \\
\hline $\mathrm{CaF}_{2}-\mathrm{Al}_{2} \mathrm{O}_{3}$ & $1451^{\circ} \mathrm{C}$ & reactions \\
$\mathrm{CaO}-\mathrm{Al}_{2} \mathrm{O}_{3}$ & & \\
\hline
\end{tabular}

body, a standard x-ray diffraction technique was used with a diffractometer (Ultima IV, Rigaku Co., Japan). For the test, the sample surfaces were slightly ground. In particular, peak intensities were obtained after removing background intensities. Microstructures were observed on fractured surfaces with SEM (VEGA II LMU, TESCAN). To observe grain boundary phases, HRTEM (TECNAI 30F, FEI) and energy dispersive spectroscopy (EDS) were performed. TEM samples were prepared using FIB (focused ion beam: LYRA3, TESCAN). The thermal conductivity of each sample was calculated based on thermal diffusivity and heat capacity using laser flash technique at $298 \mathrm{~K}$.

\section{Results and Discussion}

\subsection{Developed phases during sintering}

Figure 1 shows the X-ray diffraction patterns for samples $\mathrm{S} 1$ and $\mathrm{S} 2$ sintered at $1600^{\circ} \mathrm{C}$ for $8 \mathrm{~h}$ in $\mathrm{N}_{2}$ atmosphere. AlN was the major phase, and $\mathrm{Cu}_{2} \mathrm{O}, \mathrm{Cu}$-aluminates, or Ca-aluminates appeared slightly (their peaks were not indicated because of their weak intensities). These minor phases seem to be mainly produced by eutectic reactions between the corresponding compositions. ${ }^{6,12,13)}$ Therefore, based on X-ray data, the following eutectic reactions are possible: (1) below $1200^{\circ} \mathrm{C}$, three kinds of Cu-related eutectic reactions, (2) between 1400 and $1500^{\circ} \mathrm{C}$, two kinds of Ca-related eutectic reactions, as shown in Table 2. Thus, the current systems have lower liquid formation temperatures than previous systems added with $\mathrm{CaF}_{2}$ alone ${ }^{6}$ or $\mathrm{CaF}_{2}+\mathrm{Y}_{2} \mathrm{O}_{3}{ }^{14)}$ Except for $\mathrm{Al}_{2} \mathrm{O}_{3}$, the additives have lower melting points $<1500^{\circ} \mathrm{C}$ as well, which is why the present systems were sintered at temperature much lower than $1800^{\circ} \mathrm{C}$.

\subsection{Densification}

The sintered densities of samples $\mathrm{S} 1$ and $\mathrm{S} 2$ are shown in Fig. 2. With extended time, the sample densities increased, and the density of sample S1 are higher than that of sample S2. In general, AlN with additives such as $\mathrm{CaF}_{2}$ as well as rare-earth and alkaline-earth oxides is well known as a liquid phase sintering system. ${ }^{3,5,6,11)}$ Based on the X-ray data as



Fig. 1. XRD patterns for samples $\mathrm{S} 1$ and $\mathrm{S} 2$ sintered at $1600^{\circ} \mathrm{C}$ for $8 \mathrm{~h}$ in $\mathrm{N}_{2}$ atmosphere. All major peaks (represented by the diamond symbol) indicate AlN. 




Fig. 2. Sintered densities of samples $\mathrm{S} 1$ and $\mathrm{S} 2$ sintered at $1600^{\circ} \mathrm{C}$ for various times in $\mathrm{N}_{2}$ atmosphere.

mentioned above, in the case of sample $\mathrm{S} 1$ with $\mathrm{Cu}$, there is first eutectic liquid formed at the lowest temperature by the eutectic reaction of $\mathrm{Cu}-\mathrm{Cu}_{2} \mathrm{O}\left(\mathrm{T}_{\mathrm{E}}: 1065^{\circ} \mathrm{C}\right) .{ }^{12)}$ The additional eutectic reaction in $\mathrm{S} 1$ could additionally contribute to densification, as opposed to in $\mathrm{S} 2$ with $\mathrm{CuO}$.

In liquid phase sintering, particle rearrangement and

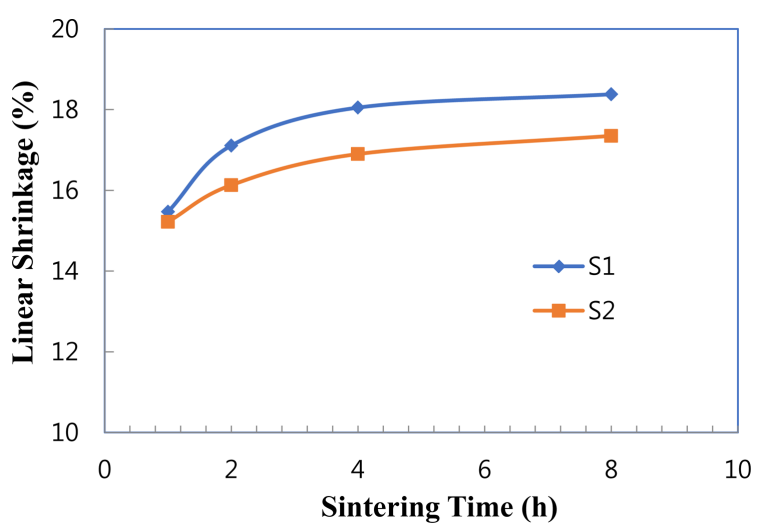

Fig. 3. Linear shrinkages of samples $\mathrm{S} 1$ and $\mathrm{S} 2$ sintered at $1600^{\circ} \mathrm{C}$ for various times in $\mathrm{N}_{2}$ atmosphere.

solution-reprecipitation of grains are the first two stages following liquid formation. In order to actively proceed with the two processes, liquid requirements such as the amount, wettability on grain surface or dihedral angle between grains, solid solubility in liquid, and solid diffusivity in liquid (liquid viscosity), are very important. In this regard, it is

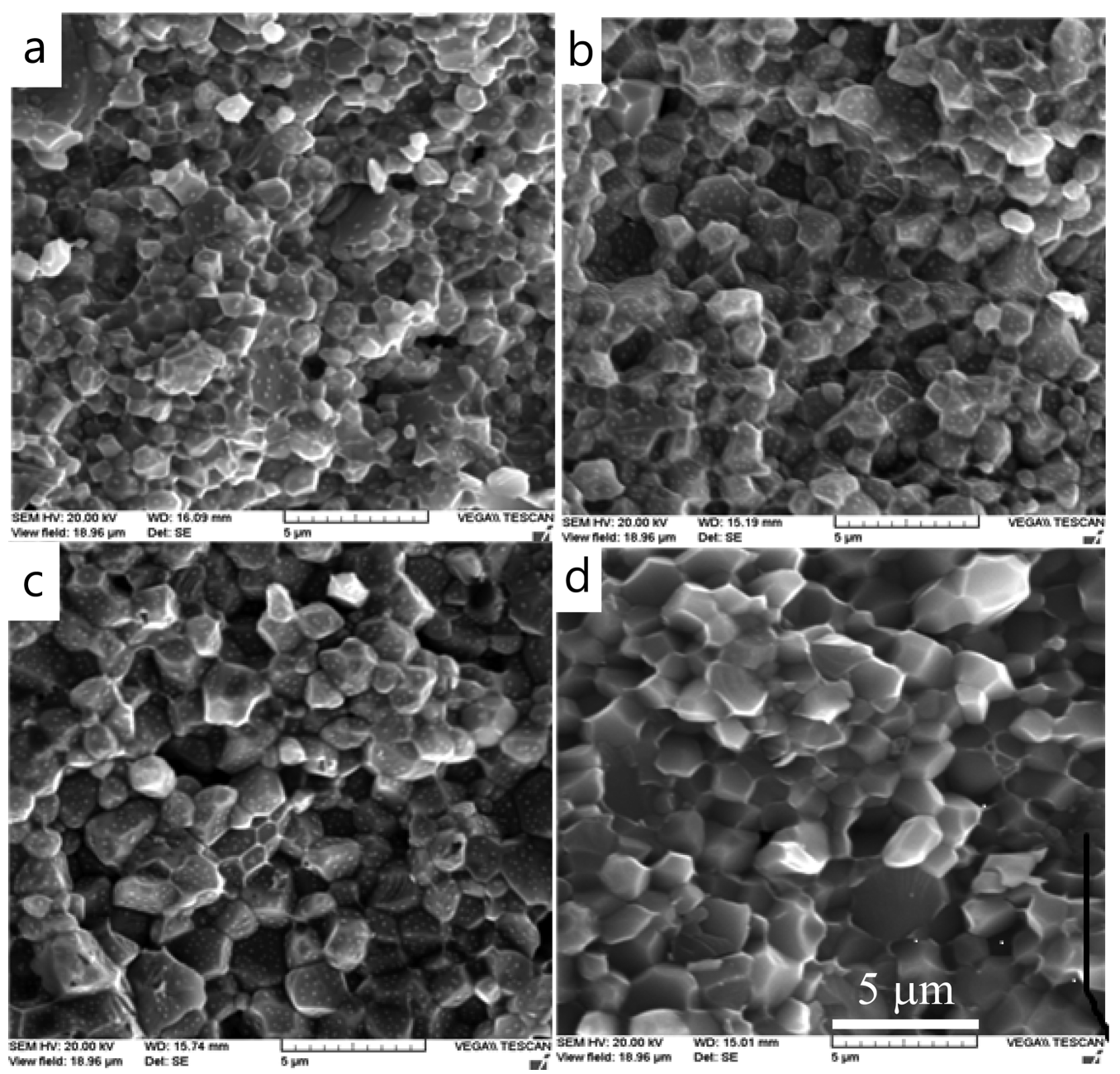

Fig. 4. SEM microstructures of the fracture surface of $\mathrm{S} 1$ sintered at $1600^{\circ} \mathrm{C}$ for various times in $\mathrm{N}_{2}$ atmosphere: (a) $1 \mathrm{~h}$, (b) $2 \mathrm{~h}$, (c) $4 \mathrm{~h}$, and (d) $8 \mathrm{~h}$. 
believed that the liquid characteristics of sample S1 are superior to those of sample S2. That is why the density for $\mathrm{S} 1$ was higher than that for S2.

Linear shrinkage showed the same trend as density, as shown in Fig. 3. With extended time, the shrinkage increased in each sample. In particular, the shrinkage in sample S1 reached up to $\sim 18.4 \%$. This value is nearly equal to that in the system with $\mathrm{Y}_{2} \mathrm{O}_{3}$ alone sintered at $1850^{\circ} \mathrm{C}$, ${ }^{7}$ and higher than that in the system with $\mathrm{CaF}_{2}+\mathrm{Y}_{2} \mathrm{O}_{3}$ altogether sintered at $1650^{\circ} \mathrm{C} .{ }^{14)}$ The current systems, thus, seem to be notable given their low sintering temperature of $1600^{\circ} \mathrm{C}$.

\subsection{Microstructure Evolution}

Figures 4 and 5 show the microstructures of the fracture surfaces of $\mathrm{S} 1$ and $\mathrm{S} 2$ sintered at $1600^{\circ} \mathrm{C}$ for various times, respectively. With prolonged time, the grain sizes increased, and the grain shapes changed from nearly spheral to hexagonal. The grain sizes for $\mathrm{S} 1$ were finer than those for S2. A liquid phase existed along the grain boundaries, as well as sphere-like things such as precipitates appeared on grain surfaces and grain boundaries for the short sintering times
(1, 2, and $4 \mathrm{~h}$ ). Based on the composition analysis, the sphere-like things were found to be migrating liquid droplets with high contact angles. That is why the liquid droplets have the same composition as the grain boundary phases. Considering that clean grain boundaries are shown on the microstructure of $8 \mathrm{~h}$ and not of $2 \mathrm{~h}$, the liquid phases seem to mostly disappear on the microstructures of $8 \mathrm{~h}$ through vaporization.

It is known that liquid phases consisting of calcium or yttrium aluminates easily vaporize at higher temperatures. ${ }^{6,74-16)}$ The liquid phases in the current systems were comprised of calcium or copper aluminates, according to the $\mathrm{X}$-ray data. As shown in Fig. 6, sample $\mathrm{S} 1$ with $\mathrm{Cu}$ showed a larger weight loss than sample $\mathrm{S} 2$ with $\mathrm{CuO}$. With prolonged time, the weight loss increased in each sample. Therefore, copper aluminates appear to be volatile at $1600^{\circ} \mathrm{C}$ as well. In this regard, it is thought that the clean grain boundaries were obtained on the microstructures of $8 \mathrm{~h}$ through the vaporization of the liquid phases. Further, the migration of the remnant liquid phases to the sample surface appears to contribute to the clean grain boundaries, as indi-

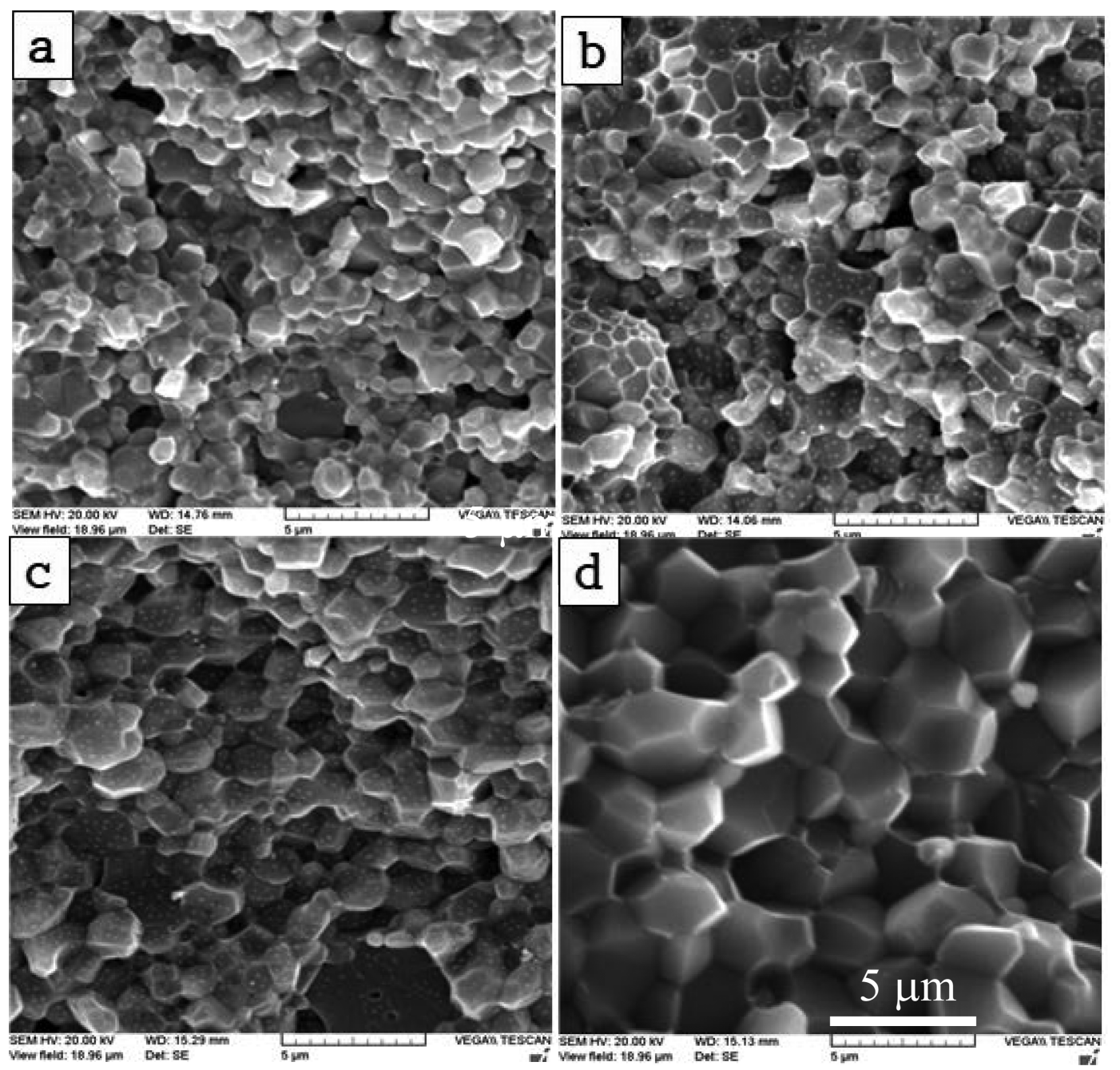

Fig. 5. SEM microstructures of the fracture surface of $\mathrm{S} 2$ sintered at $1600^{\circ} \mathrm{C}$ for various times in $\mathrm{N}_{2}$ atmosphere: (a) $1 \mathrm{~h}$, (b) $2 \mathrm{~h}$, (c) $4 \mathrm{~h}$, and (d) $8 \mathrm{~h}$. 


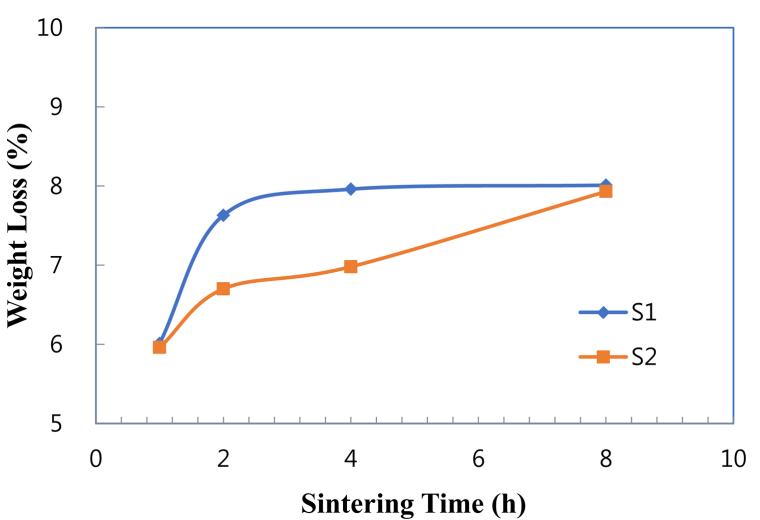

Fig. 6. Weight losses of samples sintered at $1600^{\circ} \mathrm{C}$ for various times in $\mathrm{N}_{2}$ atmosphere.

cated in the previous studies. ${ }^{11,16)}$

Typically, grain growth and grain shape changes occur simultaneously during solution-reprecipitation, which results in the smaller grains dissolving and the larger grains growing in a liquid phase. Based on the LSW theory, ${ }^{17)}$ two mechanisms, called diffusion-controlled and interface-controlled for atom movement, are involved in grain growth and shape change. Each mechanism proceeds through two steps called atom diffusion in liquid and atom attachment on a larger grain surface. During this process, the rate-determining step is the slowest one among them. Therefore, the ratedetermining step for the diffusion-controlled growth is the diffusion process, and the rate-determining step for the interface-controlled growth is the attachment process. The distinction between the two mechanisms is whether the growth rate depends on the liquid amount as well as whether the grain shape has a facet or not. In general, the grain shape for the diffusion-controlled growth appears as an equiaxed shape without elongated facets. As shown in Figs. 4 and 5, grains had the equiaxed shape with extended sintering time. It is thus believed that the grain growth was controlled by the diffusion process for the current systems. Thus, the amount of liquid seems to be an affecting factor for the grain size difference between samples $\mathrm{S} 1$ and $\mathrm{S} 2$. This result is also well supported by previous research. ${ }^{6}$ )

\subsection{Grain Boundary Phase}

Figure 7 shows TEM micrographs of sample S1 sintered

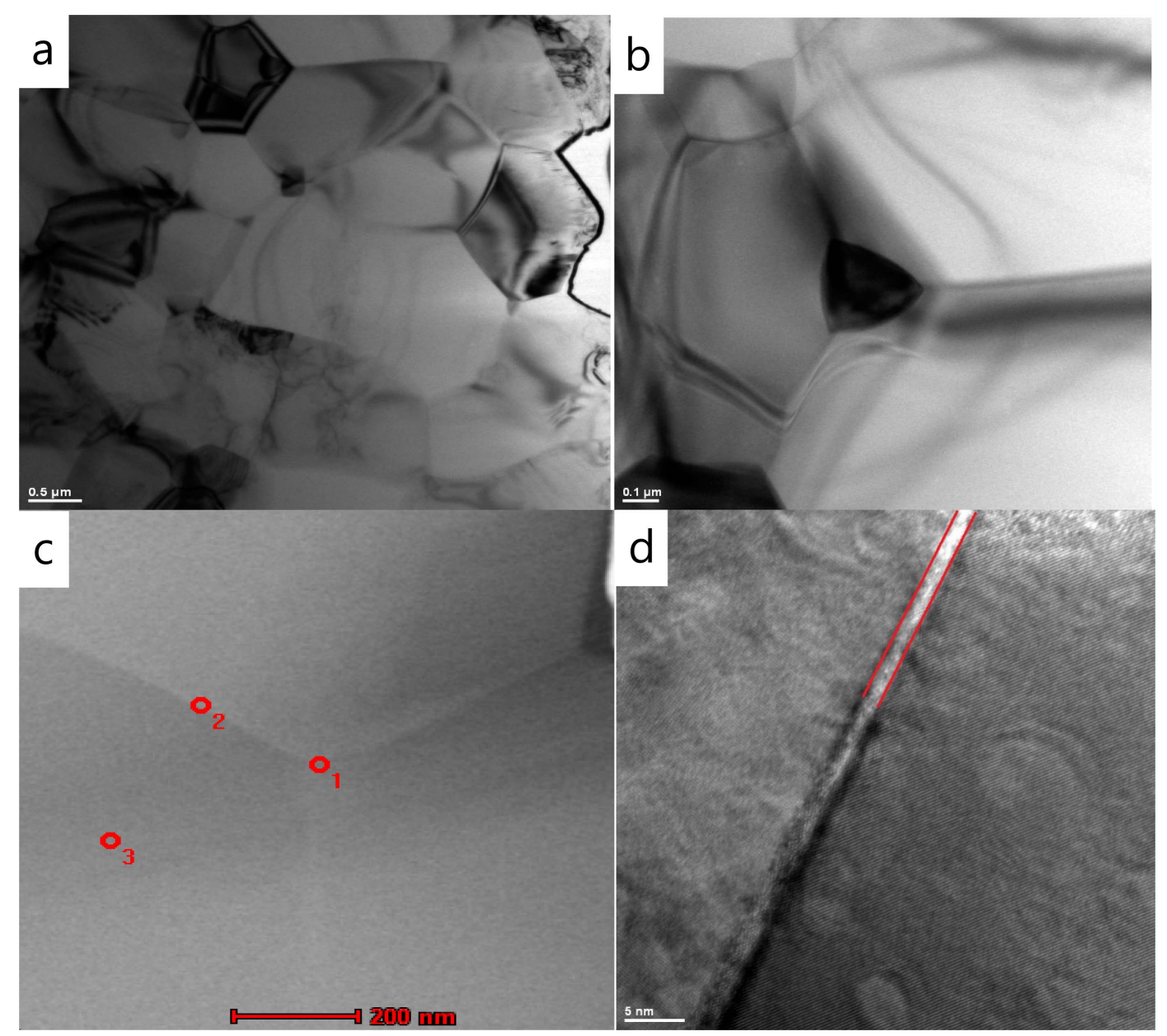

Fig. 7. TEM micrographs of sample $\mathrm{S} 1$ sintered at $1600^{\circ} \mathrm{C}$ for $8 \mathrm{~h}$ in $\mathrm{N}_{2}$ atmosphere: (a) low magnification image, (b) image showing triple junctions, (c) image showing clean grain boundaries, and (d) HRTEM image showing an intergranular amorphous film at a grain boundary. 
Table 3. Elemental Compositions at a Triple Junction, a Grain Boundary, and the Inside of a Grain

(atomic \%)

\begin{tabular}{cccc}
\hline Element & Point 1 & Point 2 & Point 3 \\
\hline $\mathrm{Al}$ & 44.75 & 76.38 & 74.55 \\
$\mathrm{~N}$ & 34.50 & 19.47 & 24.71 \\
$\mathrm{Ca}$ & 2.83 & 0.33 & 0.00 \\
$\mathrm{Cu}$ & 0.67 & 2.77 & 0.73 \\
$\mathrm{O}$ & 17.24 & 1.02 & 0.00 \\
\hline
\end{tabular}

at $1600^{\circ} \mathrm{C}$ for $8 \mathrm{~h}$. It can be seen in the TEM micrograph ((a)) that the grains were nearly equiaxed without an elongated facet, which is identical to the SEM microstructure of Fig. 4(e). This indicates that grain growth in the sample was proceeded by the diffusion-controlled mode, as mentioned above. The intergranular phases at triple grain junctions ((b)) showed a high dihedral angle $\left(>80^{\circ}\right)$, which means poor wettability for the grain surface. Due to the unfavorable wetting, the intergranular liquid phases at grain boundaries retreat to the triple junctions. With extended sintering time, the liquid phase might exude to the sample surface and then eventually vaporize, leading to its progressive thinning between grains. As a result of the exudation and vaporization, clean grain boundaries could be obtained, as shown in Fig. 7(c). The progressive thinning is supported by weight loss with sintering time, as seen in Fig. 6.

Figure 7(d) shows that the intergranular phase at the grain boundary existed as an amorphous film under $2 \mathrm{~nm}$ as a result of its progressive thinning with sintering time. The film thickness affects the thermal conductivity of AlN; this is why the thinner film showed the higher contiguity between grains. This result is supported by research on the previous systems $^{6,16)}$ sintered at higher temperatures than the present systems.

Table 3 shows elemental compositions analyzed by EDS at a triple junction, a grain boundary, and the inside of a grain, as indicated by 1, 2, and 3 on the microstructure of Fig. 7(c), respectively. As shown in Table 3, it is thought that the liquid phase was composed of $\mathrm{Cu}$ - and $\mathrm{Ca}$-aluminates, in which matrix atoms of AlN were dissolved. Characteristically, calcium was not incorporated into AlN matrix, but copper slightly dissolved in AlN matrix. As mentioned in the Introduction, oxygen impurity is detrimental to the good thermal conductivity of AlN. Thus, oxygen removal in AlN lattice is also an important consideration in selecting sintering additives. In this regard, the current system seems to remove the oxygen impurity well, given that the oxygen content is nearly zero at the inside of a grain, as seen in Table 3.

\subsection{Thermal conductivity}

The thermal conductivities of both systems of $8 \mathrm{~h}$ were measured by the laser flash technique at $298 \mathrm{~K}$. Generally, the thermal conductivity of AlN is affected by densification, grain boundary phases and oxygen contents in the lattice for the sintered body. Therefore, in view of the microstructure evolution and oxygen removal, it was expected that the thermal conductivity would be high. The measured values, however, were $\sim 70 \mathrm{~W} / \mathrm{m} \cdot \mathrm{K}$ for both systems, which is a lower value than expected. Nevertheless, considering the progressive thinning of the intergranular liquid phase which has much lower thermal conductivity ${ }^{9)}$ than AlN lattice, the thermal conductivity would be enhanced when the sintering time is increased. The reason for this is that the thinner the liquid phase is, the higher the contiguity between grains is as well.

\section{Conclusions}

AlN has a wide range of applications because of its various properties. The sintering temperature, however, is still higher than $1800^{\circ} \mathrm{C}$. Therefore, in order to reduce the manufacturing cost, the sintering temperature must be lowered. In this vein, $\mathrm{CaF}_{2}, \mathrm{CuO}$ and $\mathrm{Cu}$ were selected as sintering additives, owing to their low melting points. A sample density $>98 \%$ was obtained when the sample was sintered at $1600^{\circ} \mathrm{C}$ for $8 \mathrm{~h}$ in $\mathrm{N}_{2}$ atmosphere. The density of the sample with $\mathrm{Cu}$ was higher than that of the sample with $\mathrm{CuO}$. Based on the X-ray data, it was additionally found that $\mathrm{Cu}$ related eutectic reactions are possible below $1200^{\circ} \mathrm{C}$. Therefore, the current systems have lower liquid formation temperatures than other systems, such as $\mathrm{AlN}+\mathrm{CaF}_{2}$ or $\mathrm{AlN}+\mathrm{Y}_{2} \mathrm{O}_{3}$. For this reason, the present systems were sintered at a much lower temperature than $1800^{\circ} \mathrm{C}$. Based on the microstructures for the fracture surfaces, the grain size of the sample with $\mathrm{Cu}$ was finer than that of the sample with $\mathrm{CuO}$. In the liquid phase sintering system, grain growth mostly proceeds through the solution-reprecipitation process. Given the grain shapes on the microstructure, it is believed that the grain growth in the present systems proceeded through the diffusion-controlled mechanism. In this case, the grain size depends on the amount of existing liquid during sintering. Considering the finer grain size for the sample with $\mathrm{Cu}$ than that for the sample with $\mathrm{CuO}$, the amount of liquid for the former appears to have been larger than that for the latter. Given the high dihedral angle at triple grain junctions, the liquid of aluminates has poor wettability on the grain surfaces. Eventually, the unfavorable wetting condition appears to lead to the vaporization of the liquids. These results are evidenced by the microstructure having clean grain boundaries, as this means that a higher contiguity between grains was obtained. The result of EDS analysis indicates that oxygen impurity was well removed in AlN lattice. In this respect, it is expected that the thermal conductivities of the current systems would be improved by extending the sintering time.

\section{Acknowledgments}

This research was supported by a grant from 2017 
Research Funds of Andong National Uinversity.

\section{REFERENCES}

1. T. Kusunose and T. Sekino, "Improvement in Fracture Strength in Electrically Conductive AlN Ceramics with High Thermal Conductivity," Ceram. Int., 42 [11] 1318389 (2016).

2. J.-W. Lee, W.-J. Lee, and S.-M. Lee, "Electrical Behavior of Aluminum Nitride Ceramics Sintered with Yttrium Oxide and Titanium Oxide," J. Korean Ceram. Soc., 53 [6] 635-40 (2016).

3. H. M. Lee, K. Bharathi, and D. K. Kim, "Processing and Characterization of Aluminum Nitride Ceramics for High Thermal Conductivity," Adv. Eng. Mater., 16 [6] 655-69 (2014).

4. R. Kobayashi, Y. Nakajima, K. Mochizuki, K. Harata, T. Koto, K. Iwai, and J. Tatami, "Densification of AlN Ceramics by Spark plasma Sintering under $1550^{\circ} \mathrm{C}, "$ Adv. Powder Technol., 27 [3] 860-63 (2016).

5. H.-J. Lee, S.-W. Kim, and S.-S. Ryu, "Sintering Behavior of Aluminum Nitride Ceramics with $\mathrm{MgO}-\mathrm{CaO}-\mathrm{Al}_{2} \mathrm{O}_{3}-\mathrm{SiO}_{2}$ Glass Additive," Int. J. Refract. Met. Hard Mater., 53 4650 (2015).

6. Y. Xiong, H. Wang, and Z. Fu, "Transient Liquid-Phase Sintering of AlN with $\mathrm{CaF}_{2}$ Additive," J. Eur. Ceram. Soc., 33 [11] 2199-205 (2013).

7. A. L. Molisani, H. N. Yoshimura, and H. Goldenstein, "Sintering Mechanisms in Aluminum Nitride with Y or Ca-Containing Additive," J. Mater. Sci.: Mater. Electron., 20 [1] 1-8 (2009).

8. J. P. Holman, Heat Transfer; p. 9, McGraw-Hill, Inc., 1981.

9. M. Medraj, Y. Baik, W. T. Thomson, and R. A. L. Drew,
"Understanding AlN Sintering through Computational Thermodynamics Combined with Experimental Investigation," J. Mater. Process. Technol., 161 [3] 415-22 (2005).

10. K. F. Cai, D. S. McLachian, G. Sauti, and E. Mueller, "The Effects of Annealing on Thermal and Electrical Properties of Reaction-Bonded AlN Ceramic," Solid State Sci., 7 [8] 945-49 (2005).

11. T. B. Jackson, A. V. Virkar, K. I. More, R. B. Dinwiddie Jr., and R. A. Cutler, "High-Thermal-Conductivity Aluminum Nitride Ceramic: The Effect of Thermodynamics, Kinetic, and Microstructural Factors," J. Am. Ceram. Soc., 80 [6] 1421-35 (1997).

12. J. Hong, J.-H. Lee, Y.-N. Oh, K.-J. Cho, D.-H. Riu, S.-T. $\mathrm{Oh}$, and C.-Y. Hyun, "Direct Bonding of $\mathrm{Cu} / \mathrm{AlN}$ Using $\mathrm{Cu}$ $\mathrm{Cu}_{2} \mathrm{O}$ Eutectic Liquid," J. Korean Powder Metall. Inst., 20 [2] 114-19 (2013).

13. P. Zhang, R. Fu, Y. Tang, B. Cao, M. Fei, and Y. Yang, "Morphology of Thick Film Metallization on Aluminum Nitride Ceramics and Composition of Interface Layer," Ceram. Int., 41 [10] 13381-88 (2015).

14. L. Qiao, H. Zhou, H. Xue, and S. Wang, "Effect of $\mathrm{Y}_{2} \mathrm{O}_{3}$ on Low Temperature Sintering and Thermal Conductivity of AlN Ceramics," J. Eur. Ceram. Soc., 23 [1] 61-7 (2003).

15. Y. Liu, H. Zhou, L. Qiao, and Y. Wu, "Low-Temperature Sintering of Aluminum Nitride with $\mathrm{YF}_{3}-\mathrm{CaF}_{2}$ Binary Additive," J. Mater. Sci. Lett., 18 [9] 703-4 (1999).

16. H. Nakano, K. Watari, and K. Urabe, "Grain Boundary Phase in AlN Ceramics Fired under Reducing $\mathrm{N}_{2}$ Atmosphere with Carbon," J. Eur. Ceram. Soc., 23 [10] 176168 (2003).

17. R. M. German, Sintering Theory and Practice; pp. 293-98, John Wiley \& Sons, Inc., 1996. 